\title{
«Quando Dio deciderà di redimere Israele»: vera e falsa Redenzione nel pensiero di Isaac Orobio de Castro
}

\author{
Myriam Silvera* \\ Università degli Studi di Roma "Tor Vergata" \\ ORCID ID: http://orcid.org/0000-0002-6184-2979
}

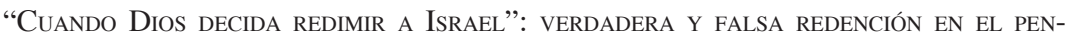
SAMIENTO DE IsAaC OROBio de CASTRO.- El presente artículo examina algunos aspectos, nunca antes estudiados, del pensamiento mesiánico de Isaac Orobio de Castro y destaca la profunda influencia que Maimónides, por un lado, y su polémica anticristiana, por otro, ejercen en el tratamiento de esta materia. A modo de ejemplo, se sigue la objeción del autor a la tesis de que el regreso a Jerusalén bajo Ciro y la Construcción del Segundo Santuario hubieran representado la definitiva realización de las esperanzas hebraicas de bienestar temporal, tesis que liquidaba para siempre una espera orientada hacia el futuro. Eso mismo había sostenido Jerónimo de Santa Fe en Tortosa y eso mismo sostenía todavía Philipp Van Limborch, contemporáneo interlocutor de Orobio. Respondiendo a esta "falsa" redención, Orobio precisa las características y los tiempos de la verdadera «esperança» del pueblo hebreo.
\end{abstract}

Palabras Clave: Philip Van Limborch; Disputa de Tortosa; polémica judía anticristiana, regreso de Babilonia; Ley de Moisés; mesianismo y literatura profética; mesianismo y Maimónides.

“When God Will Redeem Israel." True and Untrue Redemption in Isaac Orobio de CAStro's Thought.- In the article some previously ignored aspects of Isaac Orobio de Castro's messianic thought are investigated as well as the strong influence by Maimonides on one hand and the anti-Christian arguments on the other. To exemplify we analyze the author's refutation of the thesis that the Jews' return to Jerusalem and the building of the second Temple would constitute the fulfillment of the Jewish expectations of a temporal well-being. Such thesis would reject in a definitive way any

msilvera@alice.it

Copyright: () 2017 CSIC. This is an open-access article distributed under the terms of the Creative Commons Attribution (CC-by) Spain 3.0 License. 
idea of expectations oriented towards the future. This was the view espoused earlier by Girolamo de Santa Fe in Tortosa and by Philip Van Limborch at the time of Orobio. In rebutting this "false" redemption, Orobio spells out the features and times of the true "esperança" of the Jewish people.

KeYwords: Philip Van Limborch; Tortosa Disputation; Jewish anti-christian polemics; Return from Babylon; Moses' Law; Messianism and prophetic literature; Messianism and Maimonides.

\section{Che tipo di ATtesa? L'influenZa maimonidea}

Non sappiamo quale posizione avesse assunto Isaac Orobio de Castro nei confronti di Shabbatai Tzvì, quando le aspettative messianiche raggiunsero il loro acme; sappiamo però che a partire dalla conversione all'Islam del presunto Messia, Orobio se ne dissociò apertamente. Nel suo "scetticismo" egli viene associato a Isaac Cardoso, un collegamento per la prima volta proposto dal fratello di quest'ultimo, Miguel Abraham Cardoso $^{1}$, uno dei più ferventi seguaci di Shabbatai, e ideologo del movimento sabbatiano $^{2}$. Dopo aver invano tentato di convincere il fratello Isaac ad aderire alla nuova fede, Miguel scriverà a un suo corrispondente, presentando i due "dottori”, già accomunati dalla pratica della medicina, uniti nel comune antisabbatianesimo:

\footnotetext{
${ }^{1}$ Yosef H. Yerushalmi, Dalla Corte al Ghetto. La vita, le opere, le peregrinazioni del marrano Cardoso nell'Europa del Seicento. Presentazione di Michele Luzzati e Michele Olivari. Traduzione di Maria Sumbulovich, revisione di Aurelio Mottola (Milano: Garzanti, 1991 [I ed. inglese 1971, II ed. 1981]) pp. 284-285, 288 e 303; Yosef KAPLAN, From Christianity to Judaism: The Story of Isaac Orobio de Castro, translated by Raphael Loewe (Oxford: Oxford University Press on behalf of The Littman Library of Jewish Civilization [1989] 2004 paperback); Yosef Kaplan, «The Attitude of the Sephardi Leadership in Amsterdam to the Sabbatian Movement, 1665-1671», in An alternative Path to Modernity. The Sephardi Diaspora in Western Europe (LeidenBoston-Köln: Brill, 2000) pp. 211-237: in particolare pp. 228-233. Kaplan non esclude la possibilità che in un primo momento Orobio fosse stato più permeabile alla propaganda del movimento, e questo sulla base di due elementi: A. Il fatto che Jacob Sasportas non lo menzioni tra gli avversari. B. La dipendenza di Orobio dal suo maestro Rephael D'Aguilar, che era tra i fedeli di Shabbatai, vedi Kaplan, From Christianity to Judaism, p. 211.

${ }^{2}$ Gershom Scholem, Šabbetay Șevi, il Messia mistico (Torino: Einaudi, 2001) passim.
} 
No se contentara el senhor Dotor Ishack Cardozo de ser un Dotor Rabenu Moseh Bar Nahman, y el Dotor Orobio de ser un dotor Rabenu Mosseh de Egipto, sino que se consiguen guente y son nefilim, y el uno se queda caido, y el otro se aparta orbo ${ }^{3}$.

Nel tralasciare per ora in questo contesto il tema dell'eventuale vicinanza di Cardoso al pensiero di Nahmanide ${ }^{4}$, non vogliamo invece accantonare la matrice maimonidea che Miguel individua nella riflessione di Orobio su Messia e messianesimo. Perché è vero che Orobio è erede della riflessione del Rambam sull'epoca messianica, ma è anche vero che, pur senza prenderne le distanze esplicitamente, non la condivide in toto. Egli ritiene con Maimonide che il Messia possa essere individuabile attraverso una serie di segni di carattere temporale e politico: sarà Re, guiderà il popolo d'Israele nella sua terra, ricostruirà il Santuario, ripristinerà i sacrifici, instaurerà la pace tra i popoli. Scriveva Maimonide in Hilkhot Melakhim XI:

In futuro sorgerà il re Messia, il quale ristabilirà il regno di Davide nella sua antica gloria, riedificherà il Tempio e riunirà i dispersi d' Israele. In quei giorni tutte le leggi torneranno ad essere applicate, come in antico: si offriranno i sacrifici, si osserveranno gli anni sabbatici e i Giubilei secondo la normativa stabilita nella Torà ${ }^{5}$.

Questi caratteri erano ripresi e condivisi da Orobio ${ }^{6}$ :

Il Messia sarà Re santissimo principe di tutti i redentori [...], riceverà il popolo confluente come un buon Pastore, governerà, giudicherà con somma giustizia e condurrà a una vita santissima con la sua dottrina $\mathrm{e}$

\footnotetext{
${ }^{3}$ Cito dalla monografia di Kaplan, p. 213, nota 16. La lettera si trova alla Bodleian Library, Opp. Add. $4^{\circ} 150$, ff. 10v-11r.

${ }^{4}$ Sul rapporto che Miguel ha invece nei confronti del pensiero di Maimonide, vedi soprattutto Nissim Yosha, Captivated by Messianic Agonies. Theology, Philosophy and Messianism in the Thought of Abraham Miguel Cardozo (Jerusalem: Yad Izhac Ben-ZviThe Hebrew University of Jerusalem, 2015) pp. 107-136, e, tra i lavori più recenti, Bruce Rosenstock, «Abraham Miguel Cardoso's messianism. A Reappraisal», AJS Review 23 (1998) pp. 63-104.

${ }^{5}$ Vedi Mishneh Torah, Hilkhot Melakhim XI; cito da Giuseppe LaRAs, Mosè Maimonide il pensiero filosofico (Brescia: Morcelliana, 1998) p. 201.

${ }^{6}$ Philip van Limborch, De Veritate Religionis Christianae Amica Collatio cum erudito Judaeo (Gouda: Justus ab Hoeve, 1687), Secundum scriptum Judaei, pp. 13-14.
} 
con il suo esempio. Siederà sul trono di suo padre Davide e disperderà i malvagi delle Genti e di Israele?

Ma Isaac Orobio non segue più le orme di Maimonide quando il Maestro esclude che l'epoca messianica possa compromettere l'ordine fisso e immutabile della natura, e alla luce di ciò invalida la possibilità di qualsivoglia deroga alle leggi naturali: «non pensare -scriveva Maimonideche il re Messia compia necessariamente miracoli e prodigi, modifichi l'assetto naturale del mondo, faccia risuscitare i morti o altre cose del genere. Niente di tutto ciò!» ${ }^{8}$.

$\mathrm{Al}$ contrario, per Orobio, che riprende il parallelo di frequente proposto nella letteratura rabbinica tra la futura Redenzione e l'esodo dall'Egitto -quando notoriamente si verificarono grandi miracoli- la futura liberazione del popolo ebraico dall'oppressione delle Nazioni straniere si accompagnerà a fenomeni almeno altrettanto prodigiosi: «Il Signore farà prodigi superiori ai miracoli d'Egitto dovunque e a favore dell'uno e dell'altro israelita, perché da là venga liberato»' .

Il sopraggiungere del miracolo per Rambam avrebbe inficiato la perfezione, l'ordine e la regolarità delle leggi di natura, sull'eternità delle quali Maimonide aveva fondato il suo concetto di eternità della Legge divina.

\footnotetext{
${ }^{7}$ Limborch, De Veritate Religionis Christianae, p. 13. Preferisco riportare nel testo la citazione tradotta in italiano sia perché la "fonte" latina citata non può in questo caso dirsi "fonte originale", visto che è riportata da Van Limborch, sia per favorire una lettura scorrevole da parte di chi non abbia familiarità con la lingua latina. Il testo latino recita: «Messias erit, utpote Rex sanctissimus, omnium redemptorum caput [...] populum venientem, ut bonus Pastor recipiet, gubernabit, rectissimè judicabit, et sua doctrina, et exemplo ad sanctissimam vitam provocabit: Sedebit in trono Davidis patris sui, ex gentibus, et ex Israële iniquos disperderet».

${ }^{8}$ Vedi nota 5. Sul pensiero di Maimonide in relazione al tema del miracolo, vedi, tra gli altri, Joseph Heller, «Maimonides' Theory of Miracles», in Between East and West. Essays dedicated to the Memory of Bela Horovitz, ed. Alexandre Altmann (London: East and West Library, 1958) pp. 112-127; Alvin J. ReINES, «Maimonides' Concept of Miracles», Hebrew Union College Annual 45 (1974) pp. 243-285; Howard KreIsel, «Miracles in Medieval Jewish Philosophy», The Jewish Quarterly Review 75 (1984) pp. 99-133; Y. Tzvi Langermann, «Maimonides and Miracles: The Growth of a (Dis)belief», Jewish History 18 (2004) pp. 147-172.

${ }^{9}$ Limborch, De Veritate Religionis Christianae, Secundum scriptum Judaei, p. 13: «Dominus in omnibus locis, et pro uno, vel altero Israëlita, prodigia efficiet, quae Aegypti miracula excedant, ut inde liberetur».
} 
Infatti, tanto la perfezione delle leggi dell'universo quanto la perfezione dei precetti della Torà veniva definita, secondo un criterio di tipo quantitativo, come ciò cui «nulla si può aggiungere e nulla si può togliere» ${ }^{10}$.

Orobio, che non condivide il principio della fissità dell'ordinamento naturale, difende però, con Maimonide, il concetto dell'eternità della Torah, che appoggia, a differenza del filosofo, alle stesse parole del testo sacro e alle raccomandazioni divine cha accompagnano la descrizione dei precetti, dell'osservanza del sabato (Es. 31,16), della pasqua (Es. 12,14), oppure, ancora, della circoncisione (Gen. 17,13). Particolare rilievo viene dato a un passo del Deuteronomio che àncora i precetti divini alla durata della vita del popolo ebraico sulla superficie terrestre:

repetidas veces en la misma Ley [el Criador] advierte a Israel su perpetuidad, su immutabilidad y eterna duración, y entre muchos lugares [...] es expreso en el Deuteronomio cap. 12 en donde, después de aver referido Moisés los divinos estatutos dice «Estos son los fueros y juicios que guardaréis para haçer en la tierra que A. Dios de tus Padres dio a ti para heredarla todos los días que vosotros fueredeis vivos sobre la tierra» ${ }^{11}$.

Attraverso questo passo il Signore avrebbe voluto proteggere Israele e prevenirlo rispetto ad altre interpretazioni del termine "sempre", che avrebbero voluto significare un tempo lungo, ma non infinito. Scrive Orobio:

\footnotetext{
${ }^{10}$ Cfr. Maimonides, Mishneh Torah, The Book of Knowledge. Edited according to the Bodleian (Oxford) Codex with Introduction, Biblical and Talmudical References, Notes and English Translation by Moses Hyamson (Jerusalem: Jerusalem Publishers, 1965) p. 44b; Hilkhot Yesode' ha-Torah 9, 1: «It is clearly and explicity set forth in the Torah that its ordinances will endure for ever without variation, diminution or addition; as it is said, "All this word which I command you, that shall ye observe to do, thou shalt not add to it, nor take away from it" (Deut. 13,1)» e, per il medesimo principio regolante le leggi dell'Universo, Mosè Marmonide, La Guida dei perplessi, a cura di Mauro Zonta (Torino: Utet, 2003) p. 416: «Tutto ciò che Dio fa sempre sarà, e ad esso non si deve aggiungere né togliere nulla $(E c c l .3,14)$. Con questo 'versetto' egli ha trasmesso il messaggio che il mondo è opera di Dio ed è perpetuo, e ha dato anche il motivo della sua perpetuità, dicendo: 'ad esso non si deve aggiungere né togliere nulla'».

${ }^{11}$ Isaac Orobio De CASTRO, Prevenciones divinas contra la vana idolatría de las Gentes, I, Edizione critica, con introduzione, note di commento e riassunti parafrasi in italiano a cura di Myriam Silvera (Firenze: Olschki, 2013) cap. 10: Prevención a Israel que la Ley es eterna, p. 58. D'ora in poi utilizzo l'abbreviazione: Prevenciones I. Nell'edizione sopra citata, come in questo saggio, ho scelto di non modernizzare la lingua spagnola, limitandomi a inserire gli accenti per agevolare la lettura.
} 
no puede en estas divinas palavras hallar duda el entendimiento israelítico, ni repuesta la malicia de la engañada Gentilidad, porque en ellas no dice el Señor que se guarde su Ley para siempre, palavra que suelen los sophísticos Gentiles interpretar «tiempo largo aunque limitado», [...] mas usó el sagrado Legislador de una frasis que no quedasse expuesta a las vanas impugnaciones del Christianismo, cuyo sentido fuesse irrefragable, diziendo «todos los días que vos fueredeis vivos sobre la tierra»: bien se ve que no hablava con solos aquellos que entonces vivían, sino con todos sus posteres y descendientes a los quales obliga a la observancia de la Ley, siempre que fueren «vivos sobre la tierra» ${ }^{12}$.

Ne risulta dunque che, se le sorti del popolo ebraico appaiono nettamente vincolate alla Legge divina e dipendenti da essa, il raggio d'azione della figura messianica viene limitato, o quantomeno circoscritto, entro l'ambito della medesima Legge.

La presentazione più radicale dell'alternativa tra Legge e Messia al fine di ottenere la salvezza viene presentata da Orobio all'inizio del dialogo con Van Limborch; qui, l'enfasi sull'osservanza dei precetti della Torah è particolarmente marcata, mentre le stesse aspettative messianiche ebraiche sono relegate ai margini del discorso.

Quando infatti Orobio chiede provocatoriamente al suo interlocutore in quale contesto dell'Antico Testamento la fede nel Messia venga prescritta come un obbligo, intende dimostrare, al contrario, che solo l'osservanza della Legge è un comandamento divino ${ }^{13}$; quando egli domanda dove, nelle Sacre Scritture, la fede nel Messia venga indicata come fonte di salvezza, intende al contrario condurre il suo interlocutore ad ammettere che la promessa della salvezza è conseguente solo all'osservanza della Legge ${ }^{14}$.

La medesima dicotomia si ripropone ancora nel contesto in cui Orobio si trova a replicare a Van Limborch, il quale aveva sostenuto che vi sono sì casi in cui la sola mancanza di fede nel Messia può essere causa di

\footnotetext{
${ }^{12}$ Prevenciones I, p. 58.

${ }^{13}$ Limborch, De Veritate Religionis Christianae, Primum scriptum Judaei, p. 1, la prima questione di Orobio era: «1. Ut assignetur locus aliquis, in quo Deus mandaverit, aut dixerit expressè, quod fides in Messiam est absolutè necessaria ad salutem generis humani, adeo ut qui non crediderit damnandus esset».

${ }^{14}$ Così, nel medesimo contesto, proseguiva la seconda domanda: «2. Ut assignetur locus, in quo Deus dixerit, quod unicum medium ad salutem Israëlis, et restitutionis in divinam gratiam, est fides in Messiam jam adventum».
} 
dannazione ed aveva esemplificato il suo discorso volgendosi al pubblico di conversos iberici di discendenza ebraica, tra i quali era presumibile vi fossero anche fautori e sostenitori della messianicità di Gesù. Costoro -si domanda- al momento dell'arrivo del Messia atteso dagli ebrei, non saranno forse respinti e puniti dal nuovo Messia perché continuano a prestar fede al Messia dei cristiani?

Non è d'accordo con me che, quando verrà il Messia che gli ebrei odierni attendono, solo gli Israeliti che lo riconoscono, saranno partecipi della redenzione e dei suoi benefici? $\mathrm{E}$ che cosa accadrà ai discendenti di stirpe ebraica, i cui genitori già hanno creduto in Gesù Cristo, discendenti che, seguendo le orme dei loro padri, credono anch'essi in Cristo e non sono più memori della loro origine da Abramo secondo la carne? Non accadrà che questi dovranno essere esclusi dai benefici del Messia, perché non avranno accolto il Messia atteso dagli odierni ebrei? ${ }^{15}$

L'argomentazione è indubbiamente singolare nel suo rappresentare un futuro per Limborch impossibile, come è altrettanto impossibile, dal suo punto di vista, l'ipotesi di un Messia diverso da Gesù Cristo.

La risposta di Orobio perseguirà la linea argomentativa che si sviluppa in tutto il dialogo: causa della salvezza del popolo ebraico è l'osservanza della legge e l'obbedienza al Creatore, causa della sua dannazione è l'abbandono della Legge di Mosè. Ergo, i conversos che avranno creduto in Gesù non parteciperanno ai benefici dell'era messianica ebraica non per il fatto di aver creduto in Gesù, cioè non per mancanza di fede nel Messia degli ebrei, ma perché non avranno osservato la volontà del Signore Iddio.

Rispondo che quando Dio deciderà di redimere Israele, tutti gli empi tra le Genti che si saranno opposti alla volontà di Dio di redimere il suo popolo, $[\ldots]$ che si rifiutino di riconoscere la Sua Mano fortissima, si

\footnotetext{
${ }^{15}$ Limborch, De Veritate Religionis Christianae, Responsio ad Primum scriptum Judaei, p. 4: «An non quaesitur agnoscat, quando Messias, quem hodierni Judaei expectant, venerit, non alios ex Israëlitis redemtionis et beneficiorum ejus fore participes, quam qui eum recipiunt $[\ldots]$ ? Quid ergo fiet tot ex genere Judaico oriundis, quorum parentes jam olim in Jesum Christum crediderunt; quique parentum suorum vestigiis insistentes, etiam in Christum credunt, nec amplius originis suae secundum carnem ex Abrahamo memores sunt? An non et illi, quoniam eum quem hodierni Judaei expectant Messiam non sunt recepturi, à beneficiis ipsius sint excludendi?».
} 
perderanno totalmente [...] Tra di loro si conteranno anche coloro che, originati da Abramo, si sono mischiati alle Genti, dimenticando la loro progenie e la Legge, i quali ormai non hanno nulla da spartire con Israele. Ma tutti costoro, periranno non perché non avranno ubbidito al Messia, ma per aver disubbidito a Dio onnipotente (corsivo mio) ${ }^{16}$.

\section{UNA FALSA REDENZIONE}

Il fatto che la Legge assuma un ruolo centrale nel pensiero di Orobio de Castro non esclude tuttavia uno spazio anche per la speranza messianica. Si tratta di un messianesimo influenzato in larga parte dalla sua polemica anticristiana, nella misura in cui la figura che per Orobio merita l'appellativo di Messia è sovente proposta in alternativa o in opposizione al Messia cristiano. Al tempo stesso l'esegesi dei passi biblici che presso i Cristiani fondano la messianicità di Gesù viene ripresa e condotta a conclusioni diverse e opposte. Così, per dimostrare che la protratta attesa ebraica ha solidi fondamenti, non essendosi ancora compiuta la realizzazione delle promesse divine, Orobio riprende contesti che hanno una lunga storia all'interno della controversia tra ebrei e cristiani. Per esempio, nelle Prevenciones divinas troviamo interi capitoli dedicati a Genesi 49,10, a Isaia 53, alle 70 settimane di Daniele ${ }^{17}$.

In questo saggio seguiremo piuttosto un tema meno frequentemente attraversato nella controversia antiebraica: la tesi secondo cui le aspettative ebraiche di redenzione spirituale e quelle di redenzione materiale si sarebbero entrambe già realizzate, seppur in tempi diversi. Nell'affrontare quest' argomento e nell'invalidare i segni della presunta e trascorsa redenzione di Israele, Orobio accentua per contrasto la sua propria visione del messianesimo.

\footnotetext{
${ }^{16}$ Limborch, De Veritate Religionis Christianae, Secundum scriptum Judaei, p. 12: «Respondeo quod quando Deus Israëlem redimere dignetur, omnes impii ex gentibus, qui voluntati Dei suum populum redimentis obstiterint, $[\ldots]$ qui ejus fortissimam manum agnoscere renuant, omnino deperdentur [...], in quibus etiam erunt ii, qui ex Abrahamo oriundi gentibus fuerunt immixti, suam progeniem, et legem obliti, qui iam cum Israële nil prorsus habent. Sed omnes isti, non quia non obedient Messiae, peribunt, sed ut Deo omnipotenti inobedientes».

${ }^{17}$ Le due ultime trattazioni ebbero anche circolazione autonoma; vedi KAPLAN, From Christianity to Judaism, pp. 249-250.
} 
L'argomentazione cui ci riferiamo ebbe molto spazio nel corso della disputa di Tortosa (febbraio 1413-novembre 1414), che vide Gerolamo de Santa Fe, assistito da diverse autorità politiche e religiose, tra cui papa Benedetto XIII, confrontarsi con una folta delegazione del rabbinato aragonese. È infatti noto che questa, di solito annoverata come la terza controversia sul Talmud -dopo Parigi e Barcellona- fu in realtà in gran parte centrata sulla figura del Messia. Nel corso di una delle numerose sessioni, la parte ebraica enumerò ben sei "condizioni" che avrebbero consentito di riconoscere l'avvento messianico: la fine della cattività e il raduno di tutti gli esiliati nella Terra Promessa, il ritorno accompagnato da miracoli eccezionali, la ricostituzione del Santuario, il ripristino dei sacrifici, il dominio del Messia sull'intero universo in un'epoca di pace per tutte le Nazioni, e, infine, lo scontro tra Gog e Magog come segno anticipatore della redenzione. Di queste condizioni, sosteneva Girolamo, alcune, che dovevano essere interpretate in senso spirituale, si erano realizzate per il tramite di Gesù Cristo, mentre le aspettative di carattere temporale si erano già compiute con il ritorno degli ebrei a Gerusalemme sotto Ciro. A quell'epoca, infatti, si sarebbero attuate quelle condizioni di benessere temporale attese dagli ebrei -per sé, per la Terra Santa, per il Santuario- e su cui essi fondavano il loro diniego della messianicità di Gesù ${ }^{18}$. Ma gli interlocutori della parte ebraica obiettarono che quel rientro non vide la confluenza a Gerusalemme del popolo proveniente da tutte le parti della terra, né esso fu accompagnato dall'indipendenza politica, come profetizzato nel Deute-

\footnotetext{
${ }^{18}$ In attesa di una ricostruzione critica dei verbali della disputa rinviamo al controverso lavoro di Antonio Pacios LóPez, La Disputa de Tortosa, vol. I: Estudio histórico-doctrinal; vol. II: Actas (Madrid-Barcelona: Inst. Arias Montano-CSIC, 1957), II, pp. 162-17, cfr. Ivi, I, p. 260. Vedi anche Yitzhac BAER, Historia de los Judios en la España christiana, trad. José Luis Lacave (Barcelona: Riopiedras ediciones [1981]) pp. 611-684; Adolph Poznanski, «Le colloque de Tortose et de San Mateo (7 février 1413-13 novembre 1414)», REJ 147 (1922) pp. 2-39, REJ 148 (1922) pp. 160-168, REJ 149 (1922) pp. 74-88, REJ 150 (1922) pp. 187-204 e REJ 151 (1923) pp. 37-46; vedi anche Gemma EscribÀ e Yom Tov Assis (eds), The Tortosa Disputation Regesta of Documents (Jerusalem: Ginzei Am Olam, 1998) in particolare l' introduzione di Yom Tov Assis; Carlos Del Valle Rodríguez, «La disputa de Tortosa: la antigua versión original hispana de las Actas», Iberia Judaica 2 (2010) pp. 203-215. Le argomentazioni sopra riportate sarebbero state presentate nel corso della ventiquattresima sessione.
} 
ronomio (30,1-5) e in Ezechiele (28,25-26) ${ }^{19}$. Girolamo tuttavia ribadiva le sue posizioni, e le rafforzava appoggiandosi all'aggettivo 'ultimo' (אחרון) che accompagna la descrizione del Secondo Santuario nelle parole del profeta Haggai $(2,7-10)^{20}$. Se per Girolamo ciò significava che le speranze ebraiche di edificazione di un Terzo Santuario non avevano più ragion d'essere, per la delegazione ebraica quell' aggettivo "ultimo" era da intendersi come "il più recente", senza con ciò escludere la possibilità dell'erezione, umana o divina, di un Santuario successivo ${ }^{21}$.

L'obiezione più articolata all'interpretazione del ritorno da Babilonia come realizzazione di gran parte delle attese messianiche ebraiche giunse da Isaac Abravanel, circa ottant'anni dopo la celebre disputa ${ }^{22}$. Secondo

\footnotetext{
${ }^{19}$ PAcios LóPez, La Disputa de Tortosa II, sessione 37, p. 283: «Respondet iudeus quod illa prophecia, sive promissio, est generalis ad quodcumque tempus in quo Israel maneret in captivitate, et illa non possumus dicere esse adimpleta in templo secundo propter diversas raciones: Primo, quoniam in illa prophecia patet qualiter Israel debebat dispergi in multis gentibus, et post, de ipsis congregari, ut patet per textum Deuteronomii, 30 cap. "Reducet te Dominus Deus tuus de captivitate tua, ac miserebitur tui, et rursum congregabit te de cunctis populis, in quos te ante dispersit". Et in templo secundo non fuerunt reducti nisi de terra Babilonie. Secundo, quoniam in dicta prophecia dicitur: "Introducet te in terram, quam possederunt patres tui, et obtinebis eam". Ex quo patet quod adhuc eam habebant possidere et obtinere ut rem propriam. Et hoc non fuit in templo secundo, quoniam semper erant subiecti regibus grecorum et imperatoribus romanis. Tercio, quoniam dicitur in dicta prophecia: "Et benedicens tibi, maioris numeri te esse faciet quam fuerunt patres tui". Et hoc eciam non fuit in templo secundo, quoniam non fuerunt de maiori numero, nec habuerunt bonum nec benediccionem, postquam subiciebantur alienigenis».

${ }^{20}$ Pacios López, La Disputa de Tortosa II, sessione 26, p. 192.

${ }^{21}$ Pacios López, La Disputa de Tortosa II, sessione 37, p. 284: «textus qui dicit "magna est gloria domus istius novissime" etc. dicit hoc propter edificacionem quam fecit Herodes in templo, quod fuit solemnius quam illud Salomonis. Et illud quod dicit "domum ultimam", non quod non debebat esse alia ulterior posteam, sed vocatur domus ultima respectu prime». Pacios López che, come attesta la controversia che ebbe con Yitzhac Baer, interviene costantemente nel suo resoconto con giudizi e commenti "di parte", definisce quest'argomentazione «bien peregrina» e «bastante descabellada» (vedi Pacios López, La Disputa de Tortosa I, pp. 142 e 261).

${ }^{22}$ Vedi Jean-Christophe AtTIAs, Isaac Abravanel. La mémoire et l'espérance, Textes choisis, traduits de l'hebréu, annotés et précedés d'une présentation (Paris: Les Editions du Cerf, 1992) in particolare pp. 215-233. Poiché non è questa la sede per citare l'ampia bibliografia di studi su Abravanel mi limito a ricordare il "classico" Benzion NeTANYAHU, Don Isaac Abravanel, statesman and philosopher (Philadelphia: The Jewish Publication Society of America, 1972).
} 
il nostro commentatore, l'epoca storica che vide parte del popolo ebraico ritornare a Gerusalemme si distingueva dall'epoca prefigurata nei testi profetici -soprattutto in Isaia- per ben 14 diverse condizioni che avrebbero dovuto prodursi con la "vera" redenzione; queste, egli aggiungeva, erano del resto già contrassegnate dalla Mano della provvidenza divina ("14" corrisponde all'ebraico yad, cfr. Ez. 37,1). Tra i "segni messianici" su cui egli insisteva maggiormente erano annoverati la vendetta sulle Nazioni e in particolare su Edom e Botzra; il raduno degli esiliati; il ritorno alla luce delle dieci tribù nascoste e la ricongiunzione di tutto Israele. La redenzione, inoltre, si sarebbe accompagnata ai medesimi segnali miracolosi che si manifestarono in Egitto, mentre il carattere più straordinario lo avrebbe assunto la resurrezione dei morti.

Orobio de Castro ripropone alcune di queste caratteristiche, o condizioni, sia nelle Prevenciones Divinas che nel De Veritate Religionis Christianae. Nelle Prevenciones l'occasione per contrastare l'interpretazione del rientro sotto Ciro come realizzazione delle promesse divine di benessere temporale è appoggiata a contesti del Deuteronomio, e a passi tratti dal libro di Isaia, Ezra e Nehemia. In Deut. 30,3-5 Dio prometteva a Israele, se pentito e osservante dei comandamenti, la restaurazione, la liberazione dai popoli in mezzo ai quali fosse stato disperso e l'intera sua ricomposizione come popolo: «Quand'anche fossero i tuoi dispersi all'estremità del cielo, di là li radunerà il Signore tuo Dio», recitava il testo biblico. Ma, come ripete Orobio, seguendo le orme della delegazione ebraica a Tortosa e di Abravanel, solo una parte del popolo rientrò a Gerusalemme e, ciò che rende più esauriente la sua confutazione, l'esodo non si verificò da più luoghi lontani l'uno dall'altro, ma dalla sola, relativamente vicina, Babilonia.

aunque ese número que refiere el Sacro Texto volvió a Jerusalem y la Santa Tierra, quedó todo el pueblo esparcido por las demás regiones del universo - scrive Orobio - ni esto es sacarlo de los quicios del cielo, pues quedava esparcido como el presente día ${ }^{23}$.

E la prova definitiva che non vi fu un raduno degli ebrei dispersi in varie parti del mondo è appoggiata alle dieci tribù, le quali non dettero allora nessun segno di voler uscire allo scoperto dal loro rifugio: «además

\footnotetext{
${ }^{23}$ Prevenciones I, cap. 22, p. 137.
} 
que los diez tribus no volvieron a quien se hizo esta promesa, como a los dos que quedaron» ${ }^{24}$.

Un'altra occasione per confutare il carattere redentivo del rientro da Babilonia è offerta ad Orobio da un contesto in cui il profeta Isaia prefigura Gerusalemme come città giusta, santa e pura e il popolo che vi ritorna pentito e purificato, circostanze queste che non avrebbero affatto accompagnato il ritorno sotto Ciro. Scrive l'Autore, citando, tra gli altri, Nicola di Lyra:

algunos de sus comentadores, como Lyra ${ }^{25}$ y otros, dicen que esta redemción y felicidad se executó en la buelta de Babilonia, reedificación de Jerusalem y de su Sagrado Templo, con numeroso exercito de Israelitas que fueron conducidos por Esdras y Zerubabel [...] Mas quan ajena e impropia de la verdad sea esta repuesta, quan impossible ajustarla a la letra del Texto Sagrado, es manifiesto a quien de propósito no quisiere engañarse, porque el Señor dice por el propheta hablando con Zión «limpiaré tus escorias y te restituiré a tu pureza [...] y serás llamada ciudad de justicia, ciudad fiel», que es lo mismo que prometer Dios que después de el castigo de los rigores quedaría Zión acrisolada y limpia de todas sus immundicias y delitos, agena de toda injusticia y livre de la infidelidad ${ }^{26}$.

Allo scopo di insistere sulla corruzione del popolo d'Israele e sulla sua mancata fedeltà ai comandamenti del Signore, le Prevenciones riprendono anche le parole di Nehemia, che condannava le gravi trasgressioni commesse a Gerusalemme persino nel giorno del sabato, quando si continuavano a eseguire lavori agricoli e pastorali (cap. 13,15 sgg).

La lista dei fattori che distinguerebbero "vera" e "falsa" redenzione si allarga anche alla constatazione della mancata indipendenza politica in quella circostanza, poiché il popolo fu sempre allora sottomesso alle potenze straniere: «en la propia patria a que se restituían eran siervos de

\footnotetext{
${ }^{24}$ Prevenciones I, cap. 22, p. 137.

${ }^{25}$ Cfr. Biblia Sacra cum glossa interlineari, ordinaria, Nicolai Lyrani expositionibus, Burgensis Additionibus, et Thoringi Replicis. Tomus quartus, Venetiis, 1588: commento di Lira a Is. 1, 25. Per una recente rilettura del celebre commentatore, $c f r$. Gilbert Dahan dir., Nicolas de Lyre, Franciscain du XIVe siècle, exégète et théologien (Troyes Mediathèque, Grand Troyes Paris: Institut d'Études Augustiniennes, 2011).

${ }^{26}$ Prevenciones II, cap. 2, copia della British Library, Harley 341, ff. 66r-v. I versetti richiamati sono Isaia 1,25-29.
} 
estraños reyes» ${ }^{27}$ scrive Orobio, sottolineando come l'alternanza al potere di regni diversi, «de todas las monarchías, de Caldeos, Persas, Arabes, Egipcios, Griegos, y Romanos ${ }^{28}$, non avesse risparmiato il popolo ebraico da sofferenze e persecuzioni.

Ancora a proposito delle differenze rispetto alla "vera" redenzione, Orobio ricorda che le promesse divine avevano ad oggetto un' epoca imperitura; a Giacobbe, per esempio, era stata promessa in eredità una terra in cui, come scrive Ezechiele $(37,25)$ :

«habitarán sus hijos e hijos de sus hijos para siempre». Lo qual -aggiunge l'autore- ahora no a acontecido, ni se cumplió en la buelta de Babilonia, pues entonces no tubieron el Messías, ni habitaron para siempre en la Santa Tierra ${ }^{29}$.

Isaia, dal canto suo, indicava la perpetuità della Redenzione come «salvación de eternidades», e prendendo a prestito tale espressione, Orobio commenta:

«Israel es salvo en A. con salvación de eternidades, ni os aregistraréis, ni os avergonçaréis hasta siempre de siempres», como si dixera: aunque en esta reducción que haré a Israel de Babilonia a su sancta patria no experimente los majores efectos de mi antigua promesa en su beneficio, aunque no vea los empeños de mi Omnipotencia como quando salió de Egipto, aunque la major parte de el pueblo quede esparcido entre las Gentes, y los restituidos a su tierra padescan persecuciones hasta que éstos últimos los desposean de el reino, y los pongan en la última miseria y captividad, no por eso desconfie Israel, ni dude de mi divina Palabra, porque es infalible y he de cumplirla dándole lo que prometí, salvándole con salvación de eternidades, con Redemción perpetua ${ }^{30}$.

Riassumendo, numerose sono le obiezioni alla tesi che vuole esaurite le speranze messianiche con la ricostruzione del Secondo Santuario e, se vi è una qualche concessione da fare, questa consiste solo nel riconoscere che quel tempo vide un parziale alleviamento delle sofferenze di Israele.

\footnotetext{
${ }^{27}$ Prevenciones II, cap. 2, f. 66.

${ }^{28}$ Prevenciones II, cap. 3, f. 67.

${ }^{29}$ Prevenciones II, cap. 18, f. 98.

${ }^{30}$ Prevenciones II, cap. 3 , f. 67. L'espressione «salvación de eternidades» è tratta da Isaia 47,15 .
} 
Es assí que permitió el Señor una cierta relaxación de la dura captividad babilónica, y reduxo parte de Israel a su antigua patria - ma, continua no fue esta redemción acompañada de los extraordinarios favores que promete a su pueblo en su futura y prometida restituición, no salió Israel con mano alta como de Egipto, no con prodigios y portentosos castigos en las Gentes y sus fabulosas deidades, no libres del ageno yugo y llenos de preciosos despojos, sino pobres, humildes, permitida su reducción por la piedad de un Rey gentílico, como vassallos tolerados, no como hijos favorecidos del infinito Poder de su Criador, [...], llenos de temores, de sobresaltos, invadidos de sus comarcanos... ${ }^{31}$

Parte delle argomentazioni respinte da Orobio nelle Prevenciones vengono ancora riaffermate e difese da Philip Van Limborch nel De Veritate Religionis Christianae. Nell'intento di vanificare il protrarsi delle speranze messianiche ebraiche, Limborch ritorna, come aveva fatto $\mathrm{Gi}$ rolamo de Santa Fe a Tortosa, a quella tesi richiamantesi alle parole del profeta Haggai, tesi secondo cui dopo il secondo Santuario costruito sotto Ciro, non ce ne sarebbe stato in futuro alcun altro ${ }^{32}$.

Questa radicale negazione delle aspettative ebraiche di redenzione doveva suonare particolarmente ostica e offensiva a Orobio che scriveva a nemmeno vent' anni di distanza dall'avventura sabbatiana e dalla conversione all'Islam di Shabbatai Tzvì.

Forse per questo motivo, come vedremo, nel rispondere a Van Limborch, Orobio non si limita a riconfermare le attese messianiche ebraiche, ma giunge sino a individuare segnali premessianici nella realtà del suo tempo. Egli viene così indotto a scrutare quanto lo circonda, offrendo, incidentalmente, anche interessanti testimonianze di carattere storico. Per esempio quando nega il carattere redentivo del rientro da Babilonia in considerazione del fatto che esso non fu preceduto dalle profonde sofferenze tradizionalmente previste, afferma che tribolazioni estreme si sono

\footnotetext{
${ }^{31}$ Prevenciones I, cap. 18, p. 109.

${ }^{32}$ Limborch, De Veritate Religionis Christianae, Responsio ad Secundum scriptum Judaei, p. 40: «Quid quod Haggaeus, cap. II, satis manifeste indicet, post templum illum secundum aliud tertium edificandum non esset; dicit quippe Deus, se templum illud repleturum gloria, qua prioris templi gloriam excedat; ac pacem in eo templo daturum; cumque hoc continget, se caelum, terram, mare et aridam commoturum; imo se hoc semel tantum facturum. Quae omnia arguunt, post illius templi excidium alterius tertii constructionem expectandam non posse».
} 
al contrario patite ai suoi giorni. Ne sarebbero un esempio le tragiche condizioni in cui versò un gruppo di nuovi cristiani portoghesi condannati a morte a Madrid nel 1632 perché accusati di aver fustigato un' effigie del Cristo (donde il nome con cui questi fatti vengono ricordati: El Cristo de la Paciencia) $)^{33}$. Un secondo segno premonitore, come abbiamo visto, era costituito dal ritorno delle dieci tribu disperse e, mentre Orobio aveva ribattuto che questo non si era verificato dopo il rientro da Babilonia, ora, invece, egli attesta la recente segnalazione di tracce del loro remoto rifugio $^{34}$.

Così per Orobio la confutazione delle presunte realizzazioni delle aspettative temporali del popolo ebraico apre la porta al presente, che sembra gravido di quelle attese mai giunte a compimento nel passato.

L'enfasi sui segni annunciatori una imminente redenzione non ci deve tuttavia trarre in inganno: non esistono nel complesso del pensiero di Orobio tensioni premessianiche, né previsione immediate di un radicale mutamento nella storia degli ebrei e dell'umanità. La sua posizione va ricondotta alla polemica con Van Limborch e con coloro che recentemente, dopo l'avventura sabbatiana, avevano trovato motivo di amplificare la loro beffa riguardo alle vane illusioni degli ebrei ${ }^{35}$. Sempre lo scopo

\footnotetext{
${ }^{33}$ Accurata descrizione degli avvenimenti in Yosef H. Yerushalmi, Dalla Corte al Ghetto, pp. 104-132.

${ }^{34}$ Limborch, De Veritate Religionis Christianae, Tertium scriptum Judaei, p. 100: «Scio [...] quod in toto Oriente, et in vastissimis Indiae regionibus, atque etiam in Ethyopia, innumeras habent Israëlitae Synagogas $[. .$.$] à quibus non à sex annis amplissimas sacro$ idiomate literas accepimus, valde miratis, quod in his regionibus etiam extarent Judaei, seu Israëlitae, ut ipsi loquuntur, qui de Christiana religione vel nullam habent notitiam, vel saltem vagam, confusam. Hos ex decem tribubus esse, suspicamur». Le lettere in ebraico citate da Orobio meriterebbero di essere meglio identificate. Si deve aggiungere che l'argomento della diaspora delle dieci tribù è utilizzato in questo stesso contesto a testimoniare un esilio precedente il rifiuto ebraico a Gesù e dunque del tutto indipendente da questa tradizionale imputazione.

${ }^{35}$ D'altronde lo stesso Van Limborch fa cenno a diversi "falsi Messia", includendo anche Shabbatai Tzvì, vedi De Veritate Religionis Christianae, Responsio ad Tertium scriptum Judaei, p. 163: «Barchochebas se venditavit esse Messiam; habebat prophetam Aquibam, qui multa jactabat miracula: plurimi credulitate vana in initio decepti sunt, sed illis profligatis ac fraude detecta ab omnibus fuere deserti. Et similis omnium impostorum, quos in initio Judaei apertis ulnis avide exceperunt, exitus fuit, etiam nuperi ridiculi Messiae Sabetha Sevi. Quis nunc illi adhaeret? ne unus quidem».
} 
polemico permette di inquadrare le oscillazioni che Orobio esprime nel De Veritate Religionis Christianae a proposito dello spazio del messianesimo nella tradizione ebraica: non deve dunque stupire che nella fase iniziale del dialogo il ruolo del Messia fosse stato quasi messo in ombra, al fine di salvaguardare la centralità della Legge, e che invece, nell'avanzare del dialogo, il Messia, e i segni che lo precorrono, riconquistino la scena, al fine di salvaguardare l'ebraica "esperança".

\section{RADICE INTERRA SECCA}

Il più profondo orientamento di Orobio sembra essere volto a una speranza messianica viva seppur lontana, anche in questo conforme al pensiero di Maimonide ${ }^{36}$. Si tratterebbe di un'epoca che va attesa con pazienza, ma che può essere anticipata con l'immaginazione, e descritta nei suoi particolari per sostenere la fede e far fronte alle avversità; non mancano dunque nelle Prevenciones le immagini della liberazione del popolo ebraico, del suo riscatto dall'asservimento alle nazioni e del ritorno alla terra dei padri.

Sin dal prologo del primo volume Orobio assicura i suoi lettori che il Signore, tramite il Pentateuco, ha rivolto a Israele precise istruzioni a riguardo della fede in Dio, dell'osservanza della Legge e della certezza della Redenzione ${ }^{37}$. La medesima esplicita chiarezza, precisa nel prologo del tomo secondo, è stata espressa anche nella letteratura profetica: «Dispuso el Señor que todo lo necessario a Israel para la firmeza en la Sagrada Ley y en la seguridad de su esperança fuese tan claro y patente como la misma Ley» ${ }^{38}$. Nell'insieme delle Prevenciones innumerevoli sono le occor-

\footnotetext{
${ }^{36}$ Cfr. soprattutto Mosè MaImonide, Commento alla Mishnà, Sanhedrin, introduzione al cap. X, Dodicesimo Principio, in Laras, Mosè Maimonide, pp. 229-230.

${ }^{37}$ Prevenciones I, prólogo, p. 13: «Es verdad, en que ningún fiel temeroso de Dios deve dudar, que todo aquello profético que conduce para confirmarnos en la divina crehencia, en la observancia de la Ley, en la esperança de nuestra Redención, permitió el Señor Dios que tan claro y distincto lo enunciassen los Cinco Libros y los antiguos Profetas, que nos fuesse fácil entenderlo sin duda ni necesidad de considerable especulación, y esto es meramente [f. 2] revelado sin necessidad de lo misterioso».

${ }^{38}$ Prevenciones II, prólogo, f. 64.
} 
renze di termini quali "nuestra esperança",39 "esperada redención", "futura redención" "41 "nuestra universal redención", insieme a singole espressioni come "la verdad de nuestra fiel esperança" $\mathrm{a}$ " "bien fundada esperança"

Una delle espressioni più felici della "esperança" che Orobio vuole infondere nei suoi lettori è data dal paragone del popolo ebraico con una radice affondata in una terra secca, laddove la secchezza, precisa l'autore, è relativa alla terra e non alla radice, che, potenzialmente, può sempre ridare la vita. Così il popolo ebraico attualmente in cattività e "nel deserto sterile" delle Nazioni, rifiorirà rigoglioso non appena il Signore avrà irrorato la terra di nuovo:

Compara el propheta a Israel en el bajo estado de su captividad a un ramo cuyas raíces están en tierra estéril por falta de aguas que lo vivifiquen [...] un ramo cuyas secas raíces parece imposible que reverdezcan y fructifiquen, assí dijo el Señor que pondría a Israel por sus transgresiones [...] y de este estado lo sacará en el tiempo de su redemción [...]. Dice el propheta como raíz de tierra seca, mas no dize como raíz de la tierra, y es muy digna de consideración la diferencia, no es menos que demonstrativa de la esperança de Israel, porque la raíz seca ya perdió la vida de planta y no tiene esperança de reverdercer, mas la que está en tierra seca aunque [...] paresca muerta, con el beneficio de el agua vuelve a su primer estado, [...] no le llamó el propheta raíz seca sino raíz en tierra seca a quien nunca faltó la esperança de reverdercer ${ }^{45}$.

Quando dipinge infine il progressivo realizzarsi della liberazione, Orobio non perde nemmeno in questo caso l' occasione per ribadire la sua

\footnotetext{
${ }^{39}$ Prevenciones I, cap. 16, p. 95; cap. 17, p. 103, cap. 19; p. 120; cap. 24, p. 148.

${ }^{40}$ Prevenciones I, cap. 25, p. 156. Prevenciones II, cap. 2, f. 67 "esperada y prometida"; cap. 3 , f. 68 ; cap. 6 , f. 74 ; cap. 13 , f. $87 \mathrm{v}$; cap. 16 , f. 92 v.

${ }^{41}$ Prevenciones II, cap. 4, f. 69; cap. 8, f. 78v; cap. 10, f. 80v; cap. 14, f. 88.

${ }^{42}$ Prevenciones I, cap. 18, p. 110; cap. 22, pp. 134 e 136; cap. 25, p. 155; Prevenciones II, l'espressione ricorre persino nei titoli dei capitoli 1, 3, 5 e 6 .

${ }^{43}$ Prevenciones II, cap. 20, f. 99v.

${ }^{44}$ Prevenciones II, cap. 27, f. 141.

${ }^{45}$ Prevenciones II, cap. 26, ff. 125v.; cfr. la fine della citazione: «mientras el Señor no hace estas misericordias a Israel y lo detiene en su captiverio en el desierto estéril de las Naciones está como raíz en tierra seca». L'immagine della radice in terra secca è tratta da Isaia 53,2.
} 
polemica anticristiana, questa volta alimentata dal confronto tra l'elevazione di Israele elo svilimento delle Nazioni. La scena si suddivide aquesto punto tra attori e spettatori, e da luogo a un parziale capovolgimento di ruoli: il popolo ebraico, in passato umiliato, disprezzato, mortificato, diviene oggetto dell'omaggio delle Genti. Esse, che conservano ancora il ricordo dell'infelice storia ebraica, diventano il necessario testimone di tutte le cose straordinarie che si stanno verificando sulla scena pubblica.

\section{LA MANO SULLA BOCCA}

Orobio può così indugiare sui diversi particolari che segnalano il capovolgimento della situazione, esprimendosi con un tono particolarmente colorito, grazie al sostegno della letteratura profetica e unendo alla ricca immaginazione di Isaia e di Ezechiele, la propria creatività esegetica. Uno degli esempi più efficaci di questa "sintesi" si ritrova nel commento di Isaac Orobio al "servo sofferente" del capitolo 53 di Isaia ${ }^{46}$. Poiché, come avanzato da gran parte dell'esegesi ebraica tradizionale ${ }^{47}$, per Orobio, quel "servo" è da identificarsi con il popolo ebraico, e non con la figura di Gesù, la storia delle angherie subite da Israele entra in larga misura nel suo commento. Tuttavia il discorso dell'Autore, non limitandosi al passato, preconizza i tempi futuri, e mette in scena la fine della cattività di Israele, dinanzi allo sguardo meravigliato dei Gentili. Così gli ebrei che sembravano in passato aver quasi perso le parvenze di essere umano, abbrutiti come erano, sono ora guardati con interrogativo stupore. Un tempo

\footnotetext{
${ }^{46}$ Contenuto nel secondo tomo delle Prevenciones Divinas (capp. 25 e 26) e circolante autonomamente; vedi KaPLan, From Christianity to Judaism, pp. 249-250.

${ }^{47}$ Sul tema esiste una vastissima letteratura: per una prima illustrazione dello spazio che questo capitolo ha nell'esegesi ebraica, $c f r$. Adolf NeUbauer, The fiftythird chapter of Isaiah according to the Jewish Interpreters. I. Texts edited from printed books and mss. II Translations, by S. R. Driver and A. Neubauer, with an Introduction by E. B. PusEY (New York: Ktav Publishing House, 1969 [I ed. 1877]). Com'è noto, non mancano nelle fonti ebraiche interpretazioni "personaliste" del "servo sofferente". Un esempio lo si trova presso lo stesso Miguel Cardoso che abbiamo richiamato all'inizio.
} 
passivi come i vermi, incapaci di opporre resistenza a chi li calpestava ${ }^{48}$, ora sostengono fieramente lo sguardo dell'altro e ne captano la sorpresa.

Lo sbigottimento e l'ammirazione degli spettatori di fronte all'Israele redento è rappresentato attraverso una semplice gestualità: il porsi la mano dinanzi alla bocca (Michà 7,15-16). Parrebbe che, dinanzi al prodigioso intervento divino che si sta rivelando, non ci sia più spazio per le parole, quasi che la lingua comune fosse ormai inadeguata alla nuova realtà. La mano alla bocca diviene così un atto commisurato al mistero del profondo rapporto che lega il Signore a Israele, rapporto percepito in maniera emotiva prima ancora che razionale. I brutti sentimenti sono "sospesi", in un tempo sospeso, trascorso il quale i Gentili dovranno adeguare la loro immagine degli ebrei del passato con quella del presente e dovranno sottoporre al vaglio tutto ciò che in proposito avevano sentito o pensato sino a un attimo prima. Con un'assimilazione tra il passo di Michà e Isaia 52,14 Orobio estende il mutismo dai popoli ai Re. Nel commentare dunque Isaia, egli scrive:

«Assí hará hablar Gentes muchas sobre él [il servo = Israele] cerrarán reyes su boca, que lo que no fue contado a ellos vieron, y lo que no oieron entendieron». Continúa el propheta la admiración que caerá sobre las Naciones viendo la admirable y no pensada redemción de Israel: dize los efectos de esta admiración [...] que son hablar unos engrandeciendo lo sucedido, y enmudecer otros atónitos y plasmados en el protento [sic $]^{49}$.

Accettare un così profondo capovolgimento del destino ebraico richiede una fase di transizione.

Ma la meraviglia degli astanti che Orobio dipinge così efficacemente, è al tempo stesso il riflesso del suo proprio mutamento di prospettive.

\footnotetext{
${ }^{48}$ Prevenciones II, cap. 26, f. 123: «Israel perseguido nunca se provocó a su defensa, por esso le llamó el mismo propheta [Isaías] guzano en el cap. 41, v. 14: "no temas guzano de Jacob, que eres no hombre ni figura de varón entre las Gentes", si no le oprimen como el guzano, que por su pequeñez no es capaz de resistir ni de procurar defenderse de quien le oprime, mas quando sea redimido, ya no será guzano sino varón y valeroso; assí lo promete el Señor en el capítulo y verso citado, pintando ambos estados a ojos de las Gentes: [...] lo primero en la captividad como guzano, lo segundo en la redemción como varones».

${ }^{49}$ Prevenciones II, cap. 26, f. 123. La prima reazione cui Orobio (con Isaia) fa cenno, l'ingrandire l'accaduto, mi sembra di scarso rilievo nell'insieme del discorso.
} 
Adesso, egli abbandona i toni più accidiosi, mentre si offusca e sbiadisce la sua costante percezione dei tentativi di sopraffazione e di inganno da parte del mondo dei cristiani; poiché è cessato lo sforzo proselitistico del cristianesimo, sembra divenga possibile prospettare nuove modalità di relazione con esso ${ }^{50}$.

\section{UN INCONTRO TRA I POPOLI A CONDIZIONE CHE...}

Tuttavia, anche nella nuova situazione, si dovrà partire dalla premessa che Israele redento è al centro della scena, e che le nazioni gli fanno da cornice. Infatti, se esse, come aveva profetizzato Michà, «canteranno un cantico nuovo» è perché e solo nella misura in cui il Signore avrà realizzato ciò che aveva promesso a Israele ${ }^{51}$.

Così Orobio dipinge la "salita” a Gerusalemme dei "pii tra le Nazioni", la loro celebrazione della festa di Succot, tradizionalmente aperta alle 70 nazioni del mondo:

I pii delle Nazioni [...] che adorarono il vero Dio, che favorirono la mirabile redenzione di Israele servendo il Signore e il popolo, mossi da amore e timore nei confronti del Signore, saranno partecipi dei benefici della Redenzione. I loro cuori, come quelli di Israele, verranno circoncisi affinché amino il Signore loro Dio con tutto il loro cuore, con tutta la loro anima $[. .$.$] e andranno di sabato in sabato, di anno in anno a Gerusa-$ lemme, adorando il Dio di Giacobbe e offrendo sacrifici nella festa delle capanne $^{52}$.

\footnotetext{
${ }^{50}$ Questo rapporto non riguarda ovviamente i popoli e i sovrani che avranno perseguitato Israele.

${ }^{51}$ Attraverso tale argomento Orobio vuole evidentemente confutare la visione cristiana di una redenzione senza la partecipazione ebraica. Cfr. Prevenciones II, cap. 5, f. 70v: «lo más substancial de este argumento consiste en que muestren los contrarios algún lugar en que Dios les prometa redención sin redimir a Israel, lo qual, no pudiendo hazer, quedan necessariamente combencidos que hasta la universal Redemción de Israel es impossible que ellos sean redimidos».

${ }^{52}$ Limborch, De Veritate Religionis Christianae, Secundum scriptum Judaei, pp. 12-13: «Pii ex gentibus [...] qui verum Deum coluerint, qui Israëlis mirabilem redemptionem adjuverint, Deo, et populo inservientes, Dei amore, et timore alliciti, redemptionis beneficiorum erunt participes: Eorum corda, ut Israëlis, circumcidentur, ut ament Dominum Deum suum toto corde suo, et tota anima sua [...] ibuntque de Sabatho in
} 
I privilegi della redenzione non saranno dunque esclusivi del popolo ebraico: Orobio ammette che ne possano godere le Nazioni del mondo, purché i loro cuori vengano "circoncisi" ${ }^{53}$. Il senso che egli vuol dare a quest'espressione si chiarisce nel confronto con un passo delle Prevenciones, dove Orobio precisa che i popoli giungeranno a Gerusalemme, consapevoli di aver in precedenza aderito a false credenze:

[Israel] siempre cree y a creído, que infinito número de las Gentes conocerán al Señor en aquel tiempo felice, le amarán, y le buscarán, llegándose a el pueblo de Israel, y dirán «enseñados de el Señor», y admirados de que guardó fee, y cumplió su palabra a su amado pueblo, «falsedad nos enseñaron nuestros padres» [Ger. 16,19], y de todos los pueblos acudirán con santo obsequio y admirable rendimiento a Jerusalem en la festividad de [las?] cabañas ${ }^{54}$.

Riconosciute erronee le convinzioni del passato, come prevedono le parole del profeta Geremia appena citate, i popoli denunceranno la "falsedad" degli insegnamenti paterni.

L'attenzione si concentra sui cristiani che, per partecipare ai benefici dell'era messianica, per meritare la denominazione di "pii delle Nazioni" dovranno sottoporre il loro credo e il loro culto a una metamorfosi. Se infatti è l'osservanza dei sette precetti di Noè a consentire la qualifica di "pio" ", almeno uno di questi precetti non è, secondo Orobio, rispettato dal cristiano: il divieto d'idolatria.

Israele potrà perciò accogliere i Gentili all'epoca messianica, non sulla base di un principo preesistente e comune a tutti gli uomini, ma in

Sabathum, de anno in annum in Hierusalaim, Deum Jacob forte adoratum, et sacrificia offerentes in festo tabernaculorum».

Cfr. Limborch, De Veritate Religionis Christianae, Secundum Scriptum Judaei, p. 12. La prima occorrenza della "circoncisione dei cuori" è in Deut. 10,16.

${ }^{54}$ Prevenciones II, cap. 5, f. 70.

${ }^{55}$ Sui sette precetti di Noè nelle Prevenciones, vedi volume I, cap. 27, p. 179; volume II, cap. 11, f. 82. Vedi in particolare la mia nota xi a p. 179 delle Prevenciones, dove ricordo anche che Orobio aveva rivolto a D'Aguilar un importante quesito in merito alle modalità di osservanza di questi precetti, sollecitato presumibilmente della lettura del Trattato teologico-politico di Spinoza. Su questo argomento, vedi Herman Prins SALOMon, «Baruch Spinoza, Isaac Orobio de Castro and Haham Mosseh Rephael d'Aguilar on the Noachites: a Chapter in the History of Thought», Arquivos do Centro Cultural Portugues 14 (1976) pp. 253-286. 
grazia di una nuova situazione che si sarà nel frattempo prodotta e cioè a condizione di una radicale rivisitazione della loro fede.

L'atteggiamento di Orobio nei confronti di cristiani e cristianesimo non poteva sfociare in una prospettiva universalistica "larga": il Dio "uno" che tutti i popoli adoreranno all' era messianica secondo Sofonia 3,1 dovrà essere il Dio di Israele.

E sarà Adonai uno e il suo Nome uno e tutto Israele sarà santificato, un regno di Sacerdoti per tutti i popoli. Allora il Dio d'Israele regnerà unico nel suo mondo che creò ${ }^{56}$.

Se all'epoca messianica il popolo ebraico godrà della supremazia spirituale, sul piano politico, al contrario, non sovrasterà le Nazioni, le quali continueranno a mantenere la loro esistenza separata, guidate e orientate dalla paterna autorità del Messia:

Il Messia sarà [...] re santissimo, principe di tutti i redentori [...] riceverà il popolo confluente come un buon Pastore, governerà, giudicherà con somma giustizia e condurrà una vita santissima con la sua dottrina e con il suo esempio. Siederà sul trono di suo padre Davide e disperderà $i$ malvagi delle Genti e di Israele [...] A lui tutti i re e tutti i popoli obbediranno, non perché eserciterà un imperio mondano come Alessandro o come Giulio Cesare, ma perché [...] abbraccerà tutte le Genti nell'amore di Dio e nel timore filiale ${ }^{57}$.

Una volte che tutti i popoli avranno riconosciuto che il Messia agisce come semplice inviato e che «i benefici della redenzione non vengono

\footnotetext{
${ }^{56}$ Limborch, De Veritate Religionis Christianae, Secundum scriptum Judaei, p. 13: «et erit Adonai unus, et nomen ejus unum: et totus Israël jam santificatum, Regnum Sacerdotum omnibus populis: Tunc Deus Israëlis solus regnabit in suo mundo, quem creavit».

${ }^{57}$ Limborch, De Veritate Religionis Christianae, Secundum scriptum Judaei, p. 13. La prima parte della citazione è stata già riportata supra, nota 7: «Messias erit, utpote Rex sanctissimus, omnium redemptorum caput [...] populum venientem, ut bonus Pastor recipiet, gubernabit, rectissimè judicabit, et sua doctrina, et exemplo ad sanctissimam vitam provocabit: Sedebit in trono Davidis patris sui, ex gentibus, et ex Israële iniquos disperderet [...] Cui omnes reges, et populi parebunt: non quod mundanum imperium exerceat, ut Alexander, vel Julius Caesar, sed quod [...] omnes gentes in Dei amore, et filiali timore, continebit».
} 
conferiti dal Messia, ma dal Signore onnipotente ${ }^{58}$, Isaac Orobio può infine rappresentare un'era in cui «non ci saranno più guerre, ma una pace perpetua, sino agli estremi della terra, non contesa, non divisioni, non diversità di sette, perché tale è la volontà del Signore» ${ }^{59}$.

Recibido: $27 / 10 / 2017$

Aceptado: 20/11/2017

${ }^{58}$ Limborch, De Veritate Religionis Christianae, Secundum scriptum Judaei, p. 13: «redemptionis beneficia non à Messia conferentur; sed à Deo omnipotenti».

${ }^{59}$ Limborch, De Veritate Religionis Christianae, Secundum scriptum Judaei, p. 13: «non ultra bellum sed pax perpetua usque ad fines terrae, non aemulatio, non contentio, non sectarum diversitas». 